\title{
Shape-Based Interpolation of Porous and Tortuous Binary Objects
}

\author{
Srinivasan Rajagopalan, Ronald A. Karwoski, and Richard A. Robb \\ Mayo Clinic and Foundation, Rochester, MN 55905 USA
}

\begin{abstract}
This paper presents a feature-guided approach for shape-based interpolation of porous and tortuous binary objects. The feature points derived from the boundaries of the candidate source objects are matched non-linearly. The intermediate objects are obtained by appropriately blending the warped source objects. A robust outlier-rejecting, non-linear point matching algorithm based on thin-plate splines is used for establishing the feature correspondence. The proposed scheme correctly handles objects with holes, large offsets and drastic invaginations. Preliminary results suggest that this approach could be used to significantly enhance the sparse Talairach-Tournoux brain atlas.
\end{abstract}

\section{Introduction}

Existing shape-based interpolation techniques can be broadly classified into distance transform and morphology based approaches. Both the approaches have problems handling objects with holes, large offsets and drastic invagination. Of late, featureguided shape-based interpolation have been proposed, wherein feature line-segments are automatically extracted and subsequently used to control the shape interpolation. The contour matching strategy used in this approach assumes that there are no outliers. This assumption complicates the handling of invagination. Hence a reliable outlier-rejecting, non-linear matching strategy is needed to handle the invaginations correctly.

\section{Method and Results}

As in other shape-based approaches, we segmented the given candidate slices and extracted the contours for the objects of interest. Feature points derived from the contours of the object to-be-interpolated were matched using a variant of the "thin plate spline - robust point matching" (TPS-RPM) algorithm [1], to establish the correspondence and the corresponding non-linear transformation. An adaptive TPS deformation strategy [2] was used to speed up the matching process. The distance maps of the objects of interest were warped using the deformation obtained above and suitably blended to get the desired interpolated object. Figure 1 shows some preliminary results from the implementation of the proposed algorithm. The first two rows show results from synthetic datasets wherein candidate slices have no overlaps 
and partial overlaps respectively. The last row shows the interpolation of the heavily invaginated cortical structures derived from the Talairach-Tournoux brain atlas. The first two columns shows the candidate slices. The third column shows the results obtained from standard distance transform approaches. Last column shows the results from our approach. In all cases, the simple distance-transform based approach fails in some manner, whereas our approach produces robust results.

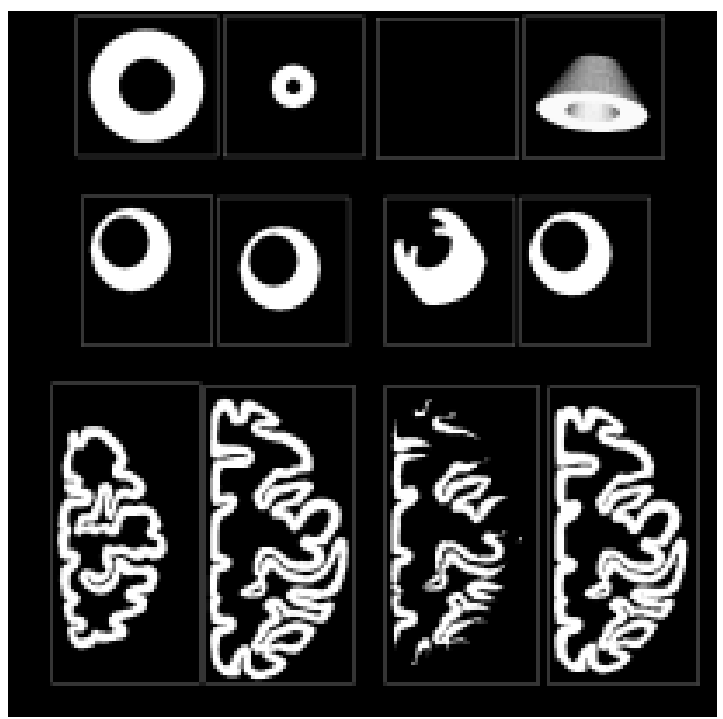

Fig. 1. Results of shape-based interpolation using simple distance-transform based algorithms and our approach

\section{Conclusion}

We have presented a feature-guided approach for shape-based interpolation of porous and tortuous binary objects. The proposed scheme correctly handles objects with holes, large offsets and drastic invagination. Preliminary results suggest that this approach could possibly be used to significantly enhance the usefulness of the sparse Talairach-Tournoux brain atlas.

\section{References}

1. H.Chui and A.Rangarajan, A New Algorithm for Non-Rigid Point Matching. In Proc. CVPR, vol. 2, pp. 40-51. 2000.

2. Shiaofen Fang, Rajagopalan Srinivasan, Raghu Raghavan, and Joan T.Richtsmeier, Volume Morphing and rendering - an integrated approach, Computer Aided Geometric Design, vol. 17, pp. 59-81, 2000. 